\title{
Recomendaciones para el examen visual en los niños
}

\author{
Vanessa López-Torres' ${ }^{1}$ Omar Fernando Salamanca-Libreros² ${ }^{2}$ Alba Lucia Törnquist ${ }^{3}$
}

\section{RESUMEN}

Introducción: el tamizaje visual en niños es una evaluación que debería ser realizada por el médico de cuidado primario para detectar disminución de la agudeza visual o factores de riesgo que puedan interferir en el desarrollo adecuado del ojo y el sistema visual. En este se debe valorar la agudeza visual, el alineamiento ocular y la presencia de anomalías oculares estructurales.

Métodos: se revisó en la literatura las estrategias para realizar un adecuado examen visual pediátrico por médicos generales y pediatras, para identificar de forma temprana los problemas visuales de los niños.

Resultados: las alteraciones visuales son una de las causas más importantes de discapacidad en los niños, cuyos factores principales de riesgo son los trastornos refractivos no corregidos, estrabismo y cataratas congénitas, identificables mediante el tamizaje visual; no detectarlas a tiempo puede dejar secuelas irreversibles.

Conclusiones: siempre que se detecte en el examen una agudeza visual por debajo de la esperada para la edad del paciente, pobre fijación y/o seguimiento después de los 3 meses, alteración del balance muscular, alteración del reflejo rojo, etc., se debe remitir el niño a un profesional de la salud visual para prevenir pérdida visual permanente.

1 Médica, Universidad San Martin, Cali. Residente de oftalmología de tercer año, Universidad del Valle, Cali, Colombia.

2 Docente de glaucoma, Universidad del Valle, Cali, Colombia.

3 Docente de optometría de la Universidad del Valle, Optómetra de la Universidad de la Salle con maestria en ciencias de la clínica optométrica de Pensylvannia College of Optometry y doctorado en neurociencias del Karolinska Institutet. Docente en la especialidad y maestría en salud ocupacional, Universidad libre de Cali y Universidad Javeriana, Cali, Colombia.

Correspondencia: Vanessa López-Torres; vanessalopez60@hotmail.com

Recibido: febrero 21 de 2018

Aceptado: julio 24 de 2018

Cómo citar: López-Torres V, Salamanca-Libreros OF, Törnquist AL. Recomendaciones para el examen visual en los niños. latreia. 2019 Ene-Mar;32(1):40-51. D0I 10.17533/udea. iatreia.v32n1a05. 


\section{PALABRAS CLAVE}

\author{
Ambliopía; Agudeza Visual; Niño; Selección visual
}

\section{SUMMARY}

\section{Recommendations for visual exam in children}

Introduction: Visual screening in children is an assessment that should be performed by the primary care physician to detect diminished visual acuity or risk factors that may interfere with an appropriate development of the eye and visual system. In the visual screening, visual acuity, ocular alignment and the presence of structural ocular anomalies should be assessed.

Methods: A literature review about strategies to perform an adequate pediatric visual examination by general physicians and pediatricians for early detection of visual problems in children was carried out.

Results: Visual disorders are one of the main causes of disability in children, being the main risk factors uncorrected refractive errors, strabismus and congenital cataracts, that can leave irreversible sequels if they are not detected on time, being those disorders identified through visual screening.

Conclusions: Whenever the visual acuity is detected below the expected for the patient's age, poor fixation and/or follow after 3 months, muscle imbalance, alteration of the red reflex, etc., the child should be referred to a visual health professional to prevent permanent visual loss.

\section{KEYWORDS}

Amblyopia; Visual Acuity; Children; Vision Screening

\section{INTRODUCCIÓN}

El tamizaje visual en los niños, es una evaluación para detectar la disminución de la agudeza visual (AV) o los factores de riesgo que puedan interferir con el desarrollo adecuado del ojo y el sistema visual. El tamizaje visual pediátrico como cuidado primario es realizado usualmente por el médico general o el pediatra en exámenes de rutina, en este se debe valorar la agudeza visual, el alineamiento ocular y la presencia de anomalías oculares estructurales, componentes indispensables para detectar problemas significativos que afectan la visión en los niños (1). Entre un 5 a 10 \% de niños preescolares presentan factores que afectan la visión, entre otros, trastornos de refracción, estrabismo, cataratas congénitas, siendo estos a su vez, las causas principales de riesgo para el desarrollo de ambliopía (2).

La ambliopía se define como la disminución de la AV por alteraciones del desarrollo de la vía visual, que no es atribuible a una enfermedad del globo ocular o del nervio óptico, con un periodo crítico en los primeros seis años de vida (3). Para un desarrollo visual normal es necesario que el cerebro reciba imágenes focalizadas y claras, pero si la imagen en la retina está distorsionada en alouno de los ojos, la imagen cortical será diferente, con lo cual el cerebro elimina la imagen menos clara produciendo ambliopía, a pesar de que el ojo se encuentre sano (4).

La sintomatología inicial de los niños con problemas visuales se puede manifestar de diversas formas, como mal rendimiento escolar, inadecuado proceso de desarrollo y dificultades psicológicas durante la adolescencia. Rara vez el niño reporta que ve mal por un solo ojo (4).

La ambliopía puede ser tratada efectivamente si se detecta antes de los 9 o 10 años. Entre más temprano se inicie el tratamiento mejores son los resultados $(5,6)$. Se ha demostrado que la detección y el tratamiento temprano de la ambliopía mejora la AV (7), así como los tamizajes visuales antes de los 37 meses disminuyen la prevalencia de ambliopía en un $60 \%$ comparado con un solo tamizaje (8). Si la ambliopía no se trata a tiempo puede producir una $A \nabla$ deficiente no corregible para el resto de la vida, con un efecto importante en la estereopsis o percepción de profundidad (6). La evidencia sugiere que después del tratamiento la $A V$ puede seguir mejorando, incluso, hasta las 12 semanas después (3).

Por lo tanto, los objetivos de este artículo de revisión son brindar información sobre las estrategias para realizar un adecuado examen visual pediátrico, identificar tempranamente problemas visuales de los niños en la consulta pediátrica, informar a los médicos de cuidado primario sobre la importancia del examen 
visual en niños y dar recomendaciones de cuándo se debe remitir el niño a un profesional de salud visual.

\section{MATERIALES Y MÉTODOS}

Se revisaron las bases de datos PubMed-Medline, Ovid y Cochrane Library utilizando los términos MeSH: Amblyopia, Visual Acuity, Children y Visual Screening. Se incluyeron artículos desde 1999 hasta el 2017 en inglés y en español.

\section{FACTORES DE RIESGO PARA DESARROLLAR AMBLIOPÍA}

Los niños con factores ambliogénicos deben ser remitidos de manera inmediata al profesional de la salud visual. Los factores de mayor riesgo son: los trastornos de refracción, cataratas congénitas y el estrabismo manifiesto, sobre todo endotropía (ET), mientras que la exotropía (XT) no confiere un riesgo significativo para desarrollar ambliopía (9).

Entre los trastornos de refracción la diferencia de poder $>1,00$ dioptría (D) entre ambos ojos, hipermetropía $>3,50 \mathrm{D}$, miopía $>3,00 \mathrm{D}$ y astiomatismo $>1,50 \mathrm{D}$ son considerados importantes factores ambliogénicos (5). Los trastornos de refracción se determinan mediante refracción bajo cicloplejia, la cual es realizada con un retinoscopio por parte del oftalmólogo u optómetra. La cicloplejia (parálisis del musculo ciliar) es necesaria para obtener una refracción más precisa en niños, debido a su tono acomodativo incrementado comparado con los adultos. El ciclopentolato al $1 \%$ es el medicamento utilizado para producir una rápida cicloplejia con duración de acción de aproximadamente 12-24 horas. Está contraindicado en caso de tener antecedente de ángulo iridocorneal estrecho e hipersensibilidad $(10,1)$.

Otras condiciones que pueden causar ambliopía son: cualquier opacidad en la córnea, cristalino, humor acuoso o humor vítreo, ptosis palpebral que ocluya el eje visual, glaucoma congénito, uveítis, retinoblastoma y retinopatía del prematuro (ROP) $(5,11-13)$. Los niños con mayor riesgo de desarrollar ROP son los menores de 2000 g de peso y/o nacidos antes de la semana 36 de edad gestacional y los niños con peso entre 2000 y 2500 g con factores de riesgo como ventilación mecánica, oxigenoterapia, corioamnionitis, sepsis neonatal por Candida, hemorragia intracraneal, leucomalacia periventricular, hidrocefalia o preclampsia severa. Estos niños deben ser examinados por un oftalmólogo con el entrenamiento correspondiente en valoración de retina de recién nacidos prematuros (14).

Los recién nacidos con edad gestacional $\leq 27$ semanas, se deben examinar al alcanzar la cuarta semana de vida y los nacidos con edad gestacional $\geq 28$ semanas, al alcanzar las 31 semanas de edad corregida o antes de salir de la unidad de cuidado intensivo, lo que se cumpla primero $(10,15)$.

Otros factores de riesgo para desarrollar la ambliopía son: ser de etnia hispana, falta de alimentación con leche materna y tabaquismo materno $(9,16)$.

\section{EPIDEMIOLOGÍA}

Según la Organización Mundial de la Salud (OMS) en menores de 15 años el número de niños con discapacidad visual es de aproximadamente 19 millones en el mundo, de los cuales 12 millones son debido a defectos refractivos y 1,4 millones de menores de 15 años, sufren de ceguera irreversible (17). De un 5 a $10 \%$ de niños preescolares tienen problemas visuales y un 5 a $7 \%$ de los que presentan trastornos de refracción requieren corrección (2). Los trastornos de la visión se encuentran dentro de las cuatro principales causas de discapacidad en Estados Unidos y son la condición más prevalente de incapacidad durante la niñez (18). En Colombia la discapacidad visual entre el 2009-2014 fue de 2.004.464 personas (19), sin embargo, a pesar de las recomendaciones de múltiples oroganizaciones, solo el $20 \%$ de los niños tienen un examen rutinario de visión (20).

Entre las condiciones que comúnmente alteran la visión se encuentran las siguientes $(10,1)$ :

Ambliopía: es la primera causa de pérdida visual en la niñez, con prevalencia de 2 a $4 \%$, incidencia de 2 a $2,5 \%$ y edad pico entre 3 y 4 años (3). Los casos de ambliopía aumentan con antecedentes familiares de este trastorno, prematuros, niños con retraso en el desarrollo y estrabismo $(10,2,20)$. Se ha demostrado que la prevalencia de ambliopía no incrementa con 
la edad en el intervalo de 5 a 15 años, sugiriendo que esta se desarrolla antes de los 5 años (21). En Colombia se ha documentado la prevalencia de ambliopía en $2,68 \%(4,22)$. Los departamentos con proporciones más altas son Cundinamarca, Nariño, Risaralda y Boyacá (19).

Estrabismo: el tipo más común de estrabismo en la población pediátrica occidental es la endotropía, en la cual el $50 \%$ de los pacientes con ET desarrollan ambliopía, afectando por igual a ambos sexos, es más común en caucásicos y afroamericanos (10).

Trastornos de refracción: es el segundo factor más contribuyente para desarrollar ambliopía después del estrabismo, siendo la hipermetropía el trastorno de refracción más común (20). Los niños con hipermetropía > 3,50 D tienen 13 veces más riesgo de desarrollar estrabismo y 6 veces más de presentar déficit de AV (5).

Cataratas congénitas: hay un estimado de 200.000 niños ciegos por catarata congénita en todo el mundo (23), con 50 a $60 \%$ de casos bilaterales $(24,25)$. En Colombia la prevalencia es 4,64 por cada 100.000 habitantes (19).

Retinopatía del prematuro (ROP): es la principal causa de ceguera infantil prevenible. En Colombia la ROP causa $10,6 \%$ de ceguera infantil $(10,15)$, con prevalencia de 3,19 por cada 100.000 habitantes (22).

Glaucoma congénito: la incidencia varía en diferentes poblaciones entre 1 en 2.500 a 1 en 68.000 personas, causando ceguera en 2 a $15 \%$ de los casos. Es bilateral en dos tercios de los afectados y entre los recién nacidos afectados se diagnostica en el $25 \%$ (10). El glaucoma congénito es más común en ciertos grupos étnicos y religiosos donde las relaciones consanguíneas son comunes. La prevalencia más alta reportada se encuentra en Arabia Saudita y en rumanos en Eslovaquia, donde se afecta 1 en 2.500 y 1 en 2.550 personas, respectivamente (26).

Uveítis: la edad media de diagnóstico es entre 8 y 9 años. $75 \%$ de casos son bilaterales. Las principales causas de ureítis en niños son idiopáticas de 25 a $54 \%$ y artritis idiopática juvenil de 15 a $47 \%(11,10)$. La prolongada duración y las complicaciones pueden causar pérdida severa de visión de 25 a 33\% de los casos (27).
Retinoblastoma: es el cáncer intraocular más común en la niñez con un caso por cada 15.000 a 20.000 nacidos vivos, representando el $2,5 \%$ a $4 \%$ de todos los cánceres pediátricos. Se diagnostican 9.000 casos nuevos cada año en el mundo y se ha encontrado mayor mortalidad en Asia y África. El retraso de más de seis meses en el diagnóstico tras el primer síntoma o signo aumenta a 70 \% la mortalidad $(28,29)$. El retinoblastoma es bilateral de 25 a $35 \%$ de los casos, siendo el primer signo leucocoria en el $56 \%$ de los casos, seguido de estrabismo en el $20 \%$ de los casos (30).

\section{EVALUACIÓN OFTALMOLÓGICA}

A continuación, se describen los pasos para realizar una adecuada evaluación oftalmológica en la consulta pediátrica (31):

\section{Historia clínica}

Es importante obtener información acerca del embarazo y periodo neonatal, problemas de salud maternos, edad gestacional, peso al nacer, desarrollo neurológico, historia familiar de estrabismo, defectos visuales o cirugías oculares previas (10). Los padres suelen ser los primeros en notar anormalidades como AV disminuida, desviaciones de los ojos e inclinación de la cabeza, entre otras (20).

El examen inicia desde que el paciente entra al consultorio observando el comportamiento visual, posiciones anormales de la cabeza, dismorfismos, habilidad para caminar, $\mathrm{y}$ demás aspectos que aporten a la evaluación. En los familiares que acompañan al paciente, se revisa la presencia de rasgos dismórficos, estrabismo, problemas oculares, etc (10).

\section{Agudeza visual y evaluación del reflejo rojo}

La Academia Americana de Oftalmología (AAO) recomienda carteles específicos para evaluar la AV de los niños según sus edades $(1,10)$ (Tabla 1).

La población pediátrica se considera entre el nacimiento y los 18 años de edad, se subdivide en 3 categorías: Infantes: desde el nacimiento hasta los 3 años, niños preescolares 3 a menos de 6 años y niños escolares 6 a 18 años (31). 
Tabla 1. Métodos apropiados por edad para el tamizaje visual pediátrico y criterios de remisión

\begin{tabular}{|c|c|c|c|c|c|c|c|}
\hline \multirow[t]{2}{*}{ Método } & \multirow[t]{2}{*}{ Indicaciones para remitir } & \multicolumn{6}{|c|}{ Edad recomendada } \\
\hline & & $\begin{array}{l}\text { Recién nacido - } 6 \\
\text { meses }\end{array}$ & $\begin{array}{c}6-12 \\
\text { meses }\end{array}$ & $\begin{array}{l}1-3 \\
\text { años }\end{array}$ & $\begin{array}{l}3-4 \\
\text { años }\end{array}$ & $\begin{array}{l}4-5 \\
\text { años }\end{array}$ & $\begin{array}{c}\text { Cada } 1 \text { - } 2 \text { años } \\
\text { después de los } \\
5 \text { años }\end{array}$ \\
\hline \multicolumn{8}{|l|}{ Agudeza visual } \\
\hline Fijación y seguimiento & Falla para fijar o seguir & $\begin{array}{c}\text { El niño coopera }>3 \\
\text { meses }\end{array}$ & - & $\bullet$ & & & \\
\hline Teller, PVE & $\begin{array}{c}\text { Teller peor de } 20 / 30, \text { PVE } \\
\text { alterados }\end{array}$ & $\begin{array}{c}\text { En el test de Teller } \\
\text { el niño coopera }>3 \\
\text { meses }\end{array}$ & $\bullet$ & $\bullet$ & & & \\
\hline LEA, HOTV & $\begin{array}{l}\text { AV peor de } 20 / 40 \text { o diferencia }> \\
2 \text { líneas de AV entre ambos ojos }\end{array}$ & & & & $\bullet$ & $\bullet$ & $\bullet$ \\
\hline Snellen, ETDRS & $\begin{array}{l}\text { AV peor de } 20 / 30 \text { o diferencia }> \\
2 \text { líneas de AV entre ambos ojos }\end{array}$ & & & & & & • \\
\hline $\begin{array}{l}\text { Reflejo Rojo } \\
\text { (Test de Bruckner) }\end{array}$ & $\begin{array}{c}\text { Ausente, blanco, opaco, falta } \\
\text { de brillo, asimétrico }\end{array}$ & $\bullet$ & $\bullet$ & $\bullet$ & $\bullet$ & $\bullet$ & $\bullet$ \\
\hline $\begin{array}{l}\text { Inspección externa } \\
\text { de ojos y párpados }\end{array}$ & $\begin{array}{l}\text { Anomalías estructurales } \\
\text { (por ejemplo ptosis) }\end{array}$ & $\bullet$ & $\bullet$ & $\bullet$ & - & $\bullet$ & $\bullet$ \\
\hline \multicolumn{8}{|l|}{ Motilidad ocular } \\
\hline Cover test & Movimiento de refijación & & & & $\bullet$ & $\bullet$ & $\bullet$ \\
\hline $\begin{array}{l}\text { Reflejo de luz corneal } \\
\text { (Test de Hirschberg) }\end{array}$ & Asimétrico o desplazado & & $\bullet$ & $\bullet$ & $\bullet$ & $\bullet$ & $\bullet$ \\
\hline Examen de pupilas & $\begin{array}{l}\text { Forma irregular, anisocoria, } \\
\text { reacción pobre o asimétrica a } \\
\text { la luz }\end{array}$ & $\bullet$ & $\bullet$ & - & - & $\bullet$ & $\bullet$ \\
\hline
\end{tabular}

Si el tamizaje no es concluyente o es insatisfactorio, el niño debe ser revalorado en 6 meses. Si en la nueva valoración no es concluyente o no puede ser realizada, se debe remitir al profesional de salud visual. PVE: Potenciales visuales evocados, AV: Agudeza visual.

Fuente: adaptado de Wallace DK, Morse CL, Melia M, Sprunger DT, Repka MX, Lee KA, et al. American Academy of Ophthalmology Preferred Practice Pattern Pediatric Ophthalmology/Strabismus Panel. Pediatric Eye Evaluations Preferred Practice Pattern ${ }^{\oplus}$ : I. Vision Screening in the Primary Care and Community Setting; Il. Comprehensive Ophthalmic Examination. Ophthalmology. 2018 Jan;125(1):P184-P227. DOI 10.1016/j.ophtha.2017.09.032.

\section{Infantes: desde el nacimiento hasta los 3 años}

Crecimiento y desarrollo del ojo: las características anatómicas oculares y fisiológicas de los niños varían ampliamente respecto a los adultos. Algunas de estas variaciones se ven reflejadas por ejemplo en la acomodación y convergencia, que están presentes a los 3 meses, en los poderes dióptricos de los lentes (córnea y cristalino) y en la AV. En un recién nacido la AV aproximada es de 20/400 la cual va mejorando en la medida en que el niño va creciendo $(32,33)$ (Tabla 2).
Antes de los tres meses de edad se puede determinar la presencia de visión anteponiendo una luz en cada ojo por separado. En caso de rechazo a la luz se considera que esta fue percibida (2).

Ambos ojos fijan entre la sexta y novena semana de vida, aunque el seguimiento de objetos puede aparecer tempranamente. La fijación, seguimiento y sostenimiento de la mirada está presente a los tres meses. El llanto o el rechazo en un niño mientras se le ocluye el ojo con mejor AV sugiere una disminución visual del ojo no ocluido (19). 


\section{Tabla 2. Características del crecimiento y desarrollo de los ojos}

Pupilas: el reflejo pupilar a la luz está presente aproximadamente a las 31 semanas de edad gestacional. Los recién nacidos tienen pupilas mióticas, las cuales van incrementando de tamaño hasta la preadolescencia.

Retina: la mácula tiene pobre desarrollo al nacimiento, con mejoría progresiva de la AV debido al aumento en la densidad foveal de los conos. La vascularización de la retina inicia en el disco óptico a las 16 semanas y alcanza la periferia (ora serrata) a las 40 semanas de gestación. La excavación del nervio óptico suele ser menor del $30 \%$.

Córnea: el diámetro corneal en recién nacidos es de 9,5 a 10,5 mm. Hacia el año de edad es de 10 a 11,5 mm, mientras que en adultos es cerca de $12 \mathrm{~mm}$. En recién nacidos y prematuros es común y normal una leve opacidad corneal, la cual resuelve en cuanto la córnea va adelgazando.

Movimientos oculares: la mirada conjugada horizontal está presente al nacimiento, mientras que la mirada vertical no es funcional hasta los seis meses de edad. Hay estrabismo intermitente en 2 de 3 bebés que se resuelve solo de 2 a 3 meses.

Las características anatómicas oculares y fisiológicas de los niños varían ampliamente respecto a los adultos. Algunas de estas variaciones se ven reflejadas en las pupilas, retina, córnea y en el desarrollo de los movimientos oculares (12).

La AV de los niños entre el nacimiento y los tres años se puede medir mediante los siguientes métodos: potenciales visuales evocados, fijación y test de mirada preferencial (Teller) $(10,34)$. De estas pruebas la más utilizada es la de fijación, en la cual se muestra un objeto llamativo al paciente, si fija, sigue y sostiene la mirada se desiona como FSS (fija sigue y sostiene). Se debe evaluar de manera binocular y monocular. Si hay pobre fijación y/o seguimiento después de tres meses de edad, se debe sospechar de una disminución de AV y referir al oftalmólogo. Debe asegurarse que el niño no esté somnoliento, ya que el desinterés o la poca cooperación pueden ocultar un problema visual $(10,31)$.

En este periodo y otras edades también es importante detectar leucocoria u opacidades en el eje visual por medio del reflejo rojo. Las causas de leucocoria pueden ser retinoblastoma, catarata, vasculatura fetal persistente, ROP, uveítis, toxocariasis, opacidad corneal, miopía alta, anisometropía, entre otros (31).

El reflejo rojo (test de Bruckner) debe ser realizado en una habitación oscura para maximizar la dilatación pupilar, con la luz irradiada en ambas pupilas simultáneamente a 1 metro de distancia. El reflejo se observa a través de un oftalmoscopio directo con las dioptrías ajustadas en cero. Debe ser rojo-amarillo brillante en cada ojo o, gris claro en ojos pigmentados de iris oscuros, debe ser idéntico en ambos ojos. Si es opaco, blanco, con falta de reflejo o asimetría son motivos de remisión al oftalmólogo $(10,31,35)$.
Esta evaluación se facilita con la dilatación pupilar, la cual tiene una baja incidencia de efectos adversos. Los medicamentos sugeridos por la AAO para dilatar a niños son tropicamida al $1 \%$ sola o, con fenilefrina al 2,5 $\%$ o Ciclopentolato al $1 \%$, aplicados 15 minutos antes del examen. Se debe evitar el uso de atropina por sus potenciales efectos adversos anticolinérgicos (36).

\section{Niños preescolares: entre 3 a menos de 6 años}

En este periodo se puede evaluar la agudeza visual con el cartel de LEA, el cual se utiliza en niños que no saben leer. Los símbolos a identificar son figuras que se muestran al niño, las cuales contrasta con las que le señala el examinador (23). Se recomienda ubicar el cartel a 3 metros de distancia (20) (Figura 1a).

EI cartel de Sheridan Gardiner o HOTV es excelente para niños que aún no leen, entre 3 y 5 años. Está compuesto por las letras $\mathrm{H}, \mathrm{O}, \mathrm{T}, \mathrm{V} \mathrm{O} \mathrm{H}, \mathrm{N}, \mathrm{S}, \mathrm{V}, \mathrm{O}$. Al paciente se le entrega un recuadro con todas las letras, mientras el examinador apunta una letra en el cartel, el niño debe identificar en el recuadro cuál es la que el examinador le está preguntando (10) (Figura $1 \mathrm{~b}$ y $1 \mathrm{c})$.

\section{Niños escolares: de 6 a 18 años}

Se usa el cartel de Snellen o ETDRS (Early Treatment Diabetic Retinopathy Study) como en los adultos. EI ETDRS es considerado como la prueba estándar para valorar la $A \nabla$ porque reúne los parámetros del comité de visión (37) (Figura 1d). 


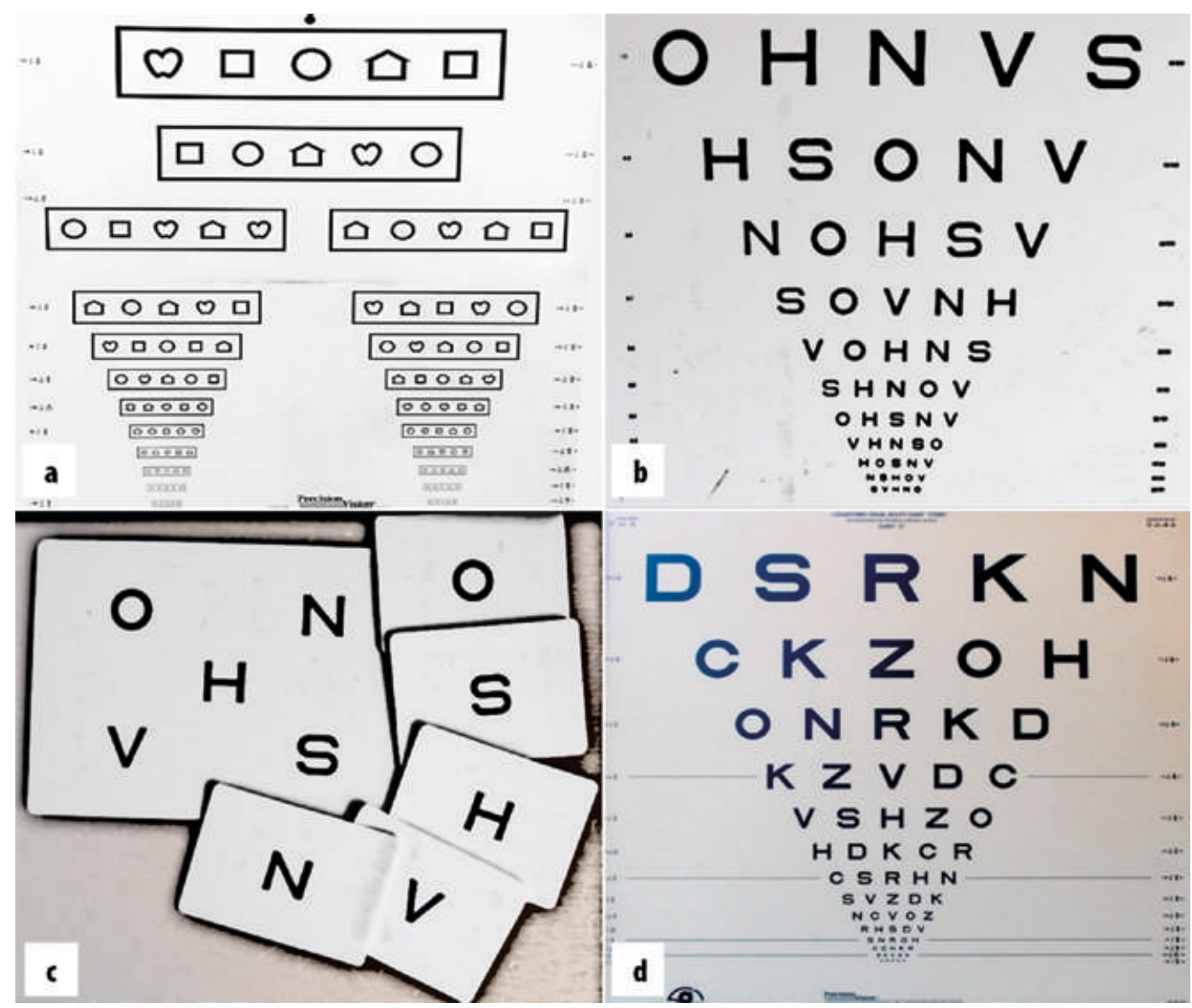

Figura 1. Carteles para la toma de agudeza visual. a. Cartel de LEA, diseñado por la doctora Lea Hyvärinen, recomendado para niños entre 3 y menos de 6 años. b y c. Carteles de Sheridan Gardiner o HOTV con sus respectivas tarjetas de comparación, recomendado para niños entre 3 y menos de 6 años. d. Cartel ETDRS (Early Treatment Diabetic Retinopathy Study), contiene 10 diferentes letras, con igual número y distanciamiento entre letras y filas e igual dificultad de los optotipos, recomendado para niños de 6 o más años $(4,12,21,37)$

Fuente: fotos propias de la autora Vanessa Lopez de carteles de consultorio para toma de agudeza visual

Los carteles para la toma de AV no siempre están disponibles en todos los consultorios, lo que a veces constituye un inconveniente para una valoración precisa de la AV. Sin embargo, se dispone de tecnologías móviles (smartphones, tablets) con aplicaciones para tomar AV. Se ha demostrado que tanto la toma de $A V$ en dispositivos electrónicos como en la cartilla de Snellen, poseen resultados comparables en ambos métodos, con excepción en AV peores de 20/200 en las que se obtuvieron mejores resultados cuando se evaluó con la cartilla de Snellen $(38,39)$.
Además de las cartillas HOTV, LEA, ETDRS y Snellen existen otras que se pueden utilizar en niños, como la de la E direccional (tumbling E), útil en mayores de 4 años. Esta consta de optotipos de legibilidad similar, pero requiere que el niño tenga orientación espacial. Otros carteles como el de Allen, Lighthouse y Kindergarten para niños de 2-4 años no están recomendados por la AAO porque no tienen legibilidad similar de los optotipos, el número de optotipos por línea es variable y no hay espacio proporcional entre estos $(7,1)$. 


\section{Niños con necesidades especiales y/o discapacidad}

La presencia de discapacidad en niños con diagnósticos como retraso del neurodesarrollo, condiciones neuropsicológicas, alteraciones del comportamiento, pueden hacer difícil la valoración de la AV usando las técnicas convencionales. Antes de iniciar el examen visual, el médico debe escoger la prueba apropiada basado en la historia clínica del paciente y aplicarla según el desarrollo mental de este (40). Se pueden usar métodos convencionales ajustados a la edad mental del paciente, como el test de Teller (1, 41, 42), HOTV (43), cartillas de Cardiff (42), fijaciónseguimiento-sostenimiento, LEA o potenciales visuales evocados (10).

\section{Inspección externa de ojos y párpados}

Examinar con linterna u oftalmoscopio directo los párpados, conjuntiva, esclera, córnea e iris. Observar si hay descarga, lagrimeo u ojo rojo que puedan deberse a una alergia, glaucoma congénito o infección, siendo la causa más común la obstrucción del ducto lagrimal, que se manifiesta en los primeros tres meses con descarga purulenta persistente de uno o ambos ojos. Si la sintomatología persiste, se debe buscar asimetría, opacidad o aumento del tamaño de la córnea y remitir al oftalmólogo. La ptosis unilateral puede causar ambliopía si cubre el eje visual, la ptosis bilateral puede estar asociada con enfermedad neurológica como miastenia $(31,44)$. Además, se debe tener en cuenta la presencia de epicanto, un pliegue de piel adicional entre los párpados que cubre la esclera nasal dando la apariencia de ET, cuando de hecho los ojos están bien alineados, condición denominada pseudoestrabismo $(6,2)$.

\section{Motilidad ocular}

El estrabismo puede ocurrir en cualquier edad y representar alteraciones en órbita, neurológicas, intraoculares o intracraneales. Tanto el reflejo corneal (test de Hirschberg) como el cover test pueden ayudar a diferenciar el estrabismo verdadero del pseudoestrabismo.
Si el examen de la motilidad ocular se encuentra alterado o hay inhabilidad para distinguir entre el pseudo $y$ estrabismo verdadero, se debe remitir $(2,31)$. Las principales formas para evaluar si hay estrabismo son:

Cover test: se basa en la observación del movimiento de refijación del ojo desviado, cuando el ojo fijador está ocluido. Las tropias se definen como un estrabismo manifiesto, mientras que las forias se definen como una tendencia al desalineamiento cuando se interrumpe la binocularidad del ojo al ocluirlo. EI cover test diferencia ambas condiciones. Este se compone del cover uncover test para las tropias y el cover test alternante para las forias. Deben ser realizados en visión lejana (3, 4 o 6 m) y cercana (33-40 $\mathrm{cm}$ ) (Figura 2a). Si el paciente ya usa corrección (gafas o lentes de contacto), se debe realizar con esta. Si es incapaz de mantener la fijación de la mirada la prueba no será confiable. La precisión del cover test para detectar desviación es mejor en niños mayores de 3 años $(6,2,45)$.

Test de Hirschberg: da un estimado del alineamiento ocular analizando el ángulo de descentramiento del reflejo corneal. Puede ser realizado a cualquier edad y es de ayuda para valorar el alineamiento ocular en niños preverbales. Se realiza con el paciente sentado y la cabeza derecha mirando hacia el frente. Se coloca una linterna a una distancia aproximada de $60 \mathrm{~cm}$ de la cara del paciente, dirigiendo la luz en el punto medio entre ambos ojos $(15,45)$. El examinador se ubica por delante del paciente y compara la posición de los dos reflejos corneales, resultando reflejos centrados o descentrados, correspondientes con ortotropia (ausencia de estrabismo) y estrabismo, respectivamente. Un reflejo centrado es aquel en el que se encuentra posicionalmente simétrico en ambos ojos, aun cuando sea fuera del centro pupilar (46). En caso de descentramiento del reflejo corneal, cada milímetro equivale aproximadamente a 7 grados de desviación del eje visual central $(14,45)$ (Figura 2b). Este test ayuda a diferenciar el estrabismo del pseudoestrabismo en caso de que la fijación sea pobre $y$ no pueda realizarse el cover test (2). 


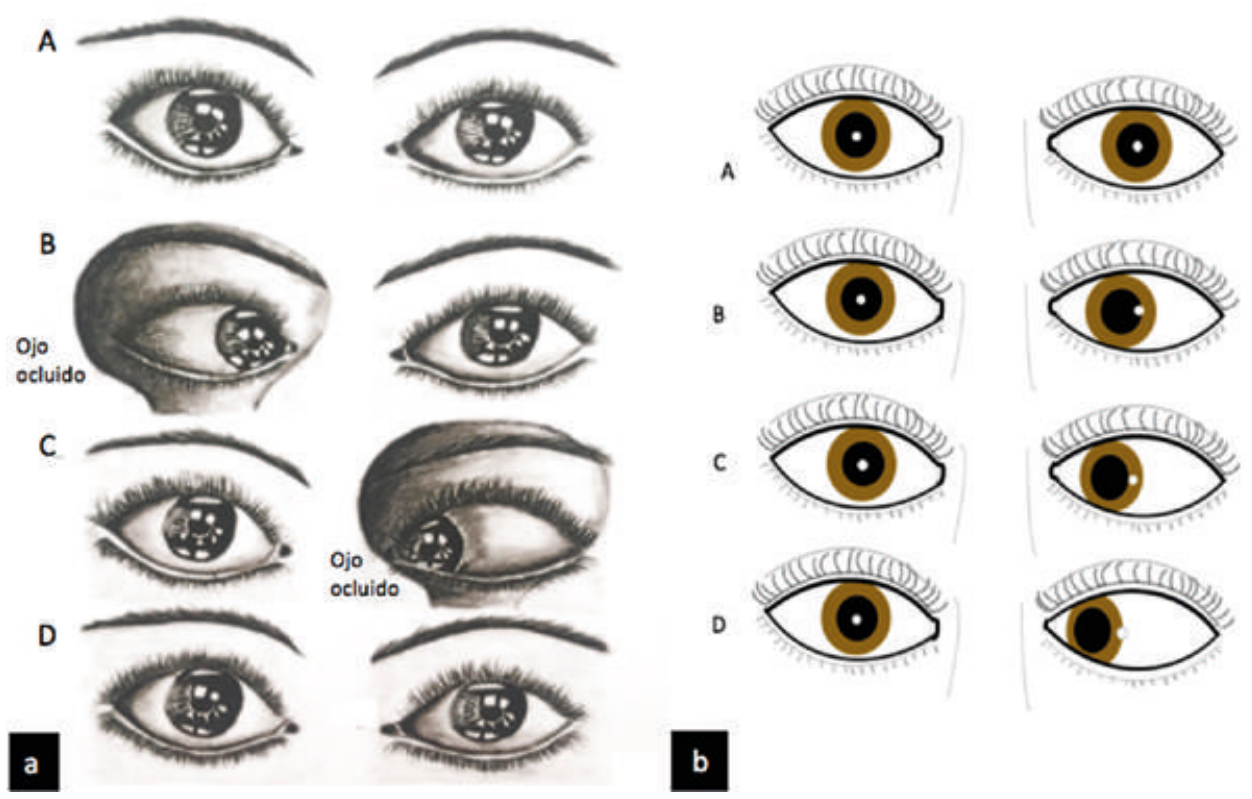

Figura 2. Pruebas para evaluar la motilidad ocular. a. Cover test. A. 0jos alineados sin desviación aparente. B. En este ejemplo al ocluir el ojo derecho se rompe la binocularidad y este se desvía hacia adentro (endodesviación), mientras que el ojo izquierdo que fija se mantiene centrado. C. Al ocluir el ojo izquierdo este se desvía hacia adentro, mientras que el ojo derecho pasa de endodesvación a estar centrado. D. Al recuperar la binocularidad se alinean ambos ojos, el ojo izquierdo que se encontraba ocluido pasa de endodesvación a estar centrado. b. Test de Hirschberg. Reflejo de luz corneal para estimación de la desviación ocular. A. Alineamiento normal. B. Reflejo de luz en el margen pupilar, endotropía izquierda (ETI) de $15^{\circ}$. C. Reflejo de luz en la mitad del iris, ETI de $30^{\circ}$. D. Reflejo de luz en el limbo, ETI de $45^{\circ}(4,12,44)$

\section{Examen de pupilas}

Deben ser redondas, reactivas e iguales. Pupilas lentas o pobremente reactivas pueden indicar disfunción retinal o del nervio óptico, por lo que se debe remitir el paciente. Se acepta como normal anisocoria hasta de $1 \mathrm{~mm}$ de diámetro. Si hay diferencia $>1 \mathrm{~mm}$ se debe sospechar de un trastorno neurológico, síndrome de Horner, parálisis del tercer par, pupila de Adie, entre otros (31). La evaluación de las pupilas en neonatos puede ser difícil por su pequeño tamaño y por la dificultad para controlar la acomodación en visión cercana (10).

\section{DISCUSIÓN}

Los trastornos de la visión se encuentran dentro de las principales causas de discapacidad en niños (18), generando un impacto económico significativo para la vida futura del paciente y su familia, con una disminución de los ingresos laborales anuales de 12 a 50 $\%$ en las personas discapacitadas que pueden trabajar, alcanzando una pérdida aproximada de 23 billones de dólares anuales entre la población afectada de los Estados Unidos (47). Los costos por tratamiento médico, aproximado para ambliopía, son de 3,6 billones de dólares en la población afectada del mismo país (48), con un costo médico por persona que varía de 400 a 3.000 dólares (49).

Por lo tanto, un adecuado examen visual pediátrico en el que se identifique si el niño requiere remisión a un profesional de salud visual, permitirá que se inicie un tratamiento temprano, que usualmente mejorará la $A V$ y la binocularidad, siendo costoefectivo (50).

Las recomendaciones para el examen visual pediátrico y los criterios de remisión sugeridos en este 
artículo están basados en las guías de la Academia Americana de Oftalmología y en el Consejo Internacional de Oftalmología. Si en el examen visual se encuentra una disminución de la $A \nabla$ según los estándares apropiados para la edad, pobre fijación y/o seguimiento con la mirada después de los 3 meses, diferencia de $\geq 2$ líneas de $A V$ entre ambos ojos, alteración del reflejo rojo, etc., se debe remitir el niño a un profesional de la salud visual, idealmente a un oftalmólogo pediatra (1).

\section{CONCLUSIONES}

El examen ocular debe realizarse al nacer y en todas las visitas deI niño sano. El no detectar los problemas visuales puede generar consecuencias como mal rendimiento académico, alteración en la interacción social, ambliopía y riesgo de ceguera bilateral si el mejor ojo se llega a lesionar. Por lo tanto, se recomienda realizar tamizaje para ambliopía antes de los 5 años y regularmente para prevenir pérdida visual permanente. Si se detectan factores de riesogo para ambliopía, se debe remitir el niño a un profesional de la saIud visual.

Cualquier profesional de la salud puede estar en capacidad de efectuar un tamizaje visual en niños, en especial los que tienen mayor contacto con la población pediátrica y un entrenamiento para realizar un adecuado examen visual pediátrico como los médicos familiares, médicos generales, en servicio social obligatorio, pediatras, ortoptistas, enfermeras y otros profesionales de la salud relacionados con el manejo de la ambliopía (22).

En Colombia la evaluación económica concluye que el tamizaje para ambliopía es costo-efectivo cuando se toma como medida de resultado en salud los casos de ambliopía evitados, independientemente de la severidad de la deficiencia en la AV. Cuando la ambliopía ya se ha establecido, el tratamiento evita una rehabilitación futura $y$ actividades especiales para individuos con baja visión (22).

\section{CONFLICTOS DE INTERESES}

Ninguno por declarar.

\section{REFERENCIAS BIBLIOGRÁFICAS}

1. American Academy of Ophthalmology. Pediatric Eye: Preferred Practice Pattern guidelines [Internet]. San Francisco: Elsevier; 2017. Available from: file:///C:/ Users/Informatica/Downloads/Pediatric\%20Eye\%20 Evaluations-FINAL-12.19.17.pdf

2. Tingley DH. Vision screening essentials: Screening today for eye disorders in the pediatric patient. Pediatr Rev. 2007;28:54-61. DOI 10.1542/pir.28-2-54.

3. Powell C, Hatt SR. Vision screening for amblyopia in childhood. Cochrane Database Syst Rev. 2009 JuI;(3):CD005020. DOI 10.1002/14651858.CD005020.pub3.

4. Parra AJ. Tamización de ambliopía en cuidado primario. Univ Med. 2009;50(2):225-36.

5. Donahue SP, Arnold RW, Ruben JB; AAPOS Vision Screening Committee. Preschool vision screening: what should we be detecting and how should we report it? Uniform guidelines for reporting results of preschool vision screening studies. J AAPOS. 2003 Oct;7(5):314-6. DOI 10.1016/S1091-8531(03)00182-4.

6. Tailor V, Balduzzi S, Hull S, Rahi J, Schmucker G, Virgili G, Dahlmann-Noor A. Tests for detecting strabismus in children age 1 to 6 years in the community. Cochrane Database Syst Rev. 2014;(7):CD011221. DOI 10.1002/14651858.CD011221.

7. Chou R, Dana T, Bougatsos C. Screening for Visual Impairment in Children Ages 1-5 Years: Systematic Review to Update the 2004 U.S. Preventive Services Task Force Recommendation [Internet]. Rockville (MD): Agency for Healthcare Research and Quality (US); 2011 Feb. Available from http://www.ncbi.nlm.nih.gov/ books/NBK52708/

8. Williams C, Northstone K, Harrad RA, Sparrow JM, Harvey I; ALSPAC Study Team. Amblyopia treatment outcomes after screening before or at age 3 years: follow up from randomised trial. BMJ. 2002 Jun;324(7353): 1549.

9. Tarczy-Hornoch K, Varma R, Cotter SA, McKean-Cowdin R, Lin JH, Borchert MS, et al. Risk factors for decreased visual acuity in preschool children: the multi-ethnic pediatric eye disease and Baltimore pediatric eye disease studies. Ophthalmology. 2011 Nor;118(11):226273. DOI 10.1016/j.ophtha.2011.06.033.

10. American Academy of Ophthalmology. Pediatric ophthalmology and clinical strabismus. BCSC: San Francisco, CA; 2016- 2017. 
11. Reiff A, Kadayifcilar S, Özen S. Rheumatic inflammatory eye diseases of childhood. Rheum Dis Clin North Am. 2013 Nov;39(4):801-32. DOI 10.1016/j. rdc.2013.05.005

12. Heiligenhaus A, Heinz C, Walscheid K, Foeldvari I. Uveitis in Children. In: Zierhut $M$, Pavesio $C$, Ohno S, Orefice F, Rao N. Intraocular inflammation. Berlin: Springer; 2016. p. 618-18. DOI 10.1007/978-3-54075387-2_48.

13. Clarke L, Guex-Crosier Y, Hofer M. Epidemiology of uveitis in children over a 10 years period. Pediatric Rheumatology. 2011;9(SuppI 1):223. DOI 10.1186/15460096-9-S1-P223.

14. Organización Panamericana de la Salud, Organización Mundial de la Salud. Guía de práctica clínica para el manejo de la retinopatía de la prematuridad [Internet]. Washington, D.C.: Pan American Health Organization; 2017 [citado Abril 2018]. Disponible en: http://iris.paho.org/xmlui/handle/123456789/34948

15. Zuluaga C, Sierra MV, Asprilla E. Causas de ceguera infantil en Cali, Colombia. Colomb Med. 2005;36(4):235-38.

16. Birch E, Castañeda Y, Wheaton D, Birch D, Uauy R, Hoffman D, et al. Visual maturation of term infants fed long-chain polyunsaturated fatty acid-supplemented or control formula for 12 mo. Am J Clin Nutr. 2005;81:871-9.

17. Organización Mundial de la Salud. Ceguera y discapacidad visual [Internet]. Ginebra: WHO; 2014 [cited 2014 Aug]. Available from: http://www.who.int/mediacentre/factsheets/fs282/es/

18. World Health Organization. Universal eye health: a global action plan 2014-2019 [Internet]. Ginebra: WHO; 2017 [cited 2017]. Available from: http://www. who.int/blindness/actionplan/en/

19. Colombia. Ministerio de Salud. Análisis de situación de salud visual en Colombia 2016 [Internet]. Bogotá: MinSalud; 2016 [citado 2016]. Disponible en:

https://www.minsalud.gov.co/sites/rid/Lists/BibliotecaDigital/RIDE/VS/PP/ENT/asis-salud-visual-colombia-2016.pdf

20. Doshi NR, Rodriguez ML. Amblyopia. Am Fam Physician. 2007 Feb;75(3):361-7.

21. Xiao O, Morgan IG, Ellwein LB, He M; Refractive Error Study in Children Study Group. Prevalence of AmbIyopia in School-Aged Children and Variations by Age,
Gender, and Ethnicity in a Multi-Country Refractive Error Study. Ophthalmology. 2015 Sep;122(9):1924-31. DOI 10.1016/j.ophtha.2015.05.034.

22. Colombia. Ministerio de Salud. Guía de práctica clínica para la prevención, la detección temprana, el diagnóstico, el tratamiento y el seguimiento de la ambliopía en menores de 18 años [Internet]. Bogotá: MinSalud; 2016 [citado 2016]. Disponible en: http:// gpc.minsalud.gov.co/gpc_sites/Repositorio/Conv_637/ GPC_ambliopia/gpc_ambliopia_completa.aspx.

23. Lloyd IC, Ashworth J, Biswas S, Abadi RV. Advances in the management of congenital and infantile cataract. Eye (Lond). 2007 Oct;21(10):1301-9.DOI 10.1038/ sj.eye. 6702845 .

24. Fan DS, Yip WWW, Yu CB, Rao SK, Lam DS. Updates on the surgical management of paediatric cataract with primary intraocular lens implantation. Ann Acad Med Singapore. 2006 Aug;35(8):564-70.

25. Trumler AA. Evaluation of pediatric cataracts and systemic disorders. Curr Opin Ophthalmol. 2011 Sep;22(5):365-79. DOI 10.1097/ICU.ob013e32834994dc.

26. Moore DB, Tomkins O, Ben-Zion I. A review of primary congenital glaucoma in the developing world. Surv Ophthalmol. 2013 May-Jun;58(3):278-85. DOI 10.1016/j.survophthal.2012.11.003.

27. Majumder PD, Biswas J. Pediatric uveitis: An update. Oman J Ophthalmol. 2013 Sep;6(3):140-50. DOI 10.4103/0974-620X.122267.

28. Dimaras H, Kimani K, Dimba EA, Gronsdahl P, White A, Chan HS. Retinoblastoma. Lancet. 2012 Apr;379(9824):1436-46. DOI 10.1016/S01406736(11)61137-9.

29. Rodriguez-Galindo C, Orbach DB, VanderVeen D. Retinoblastoma. Pediatr Clin North Am. 2015 Feb;62(1):20123. DOI 10.1016/j.pcl.2014.09.014.

30. Pandey AN. Retinoblastoma: An overview. Saudi J Ophthalmol. 2014 Oct;28(4):310-5. DOI 10.1016/j. sjopt.2013.11.001.

31. Committee on practice and ambulatory medicine on ophthalmology, American Association of Certified Orthoptists, American Association For Pediatric Ophthalmology and Strabismus, American academy of ophthalmology. Eye examination in infants, children and young adults by pediatricians. Ophthalmology. 2003;110(4):860-65. DOI 10.1016/S0161-6420(03)00414-7. 
32. American Academy of Ophthalmology. Fundamentals and principles of ophthalmology 2017-2018. Section 2 [Internet]. San Francisco CA: BCSC;2017 [citado 2016]. Available from: file://C:/Users/Informatica/Downloads/ BCSC1718_So2.pdf

33. Powell C, Wedner S, Hatt SR. Vision screening for correctable visual acuity deficits in school-age children and adolescents. Cochrane Database Syst Rev. 2005;(4):CD005023. DOI 10.1002/14651858.CD005023.pub2.

34. Leat SJ, Yadar NK, Irving EL. Development of visual acuity and contrast sensitivity in children. J Optom. 2009;2(1):19-26. DOI 10.3921/joptom.2009.19.

35. Wright K. Ocular examination and vision screening. In: Pediatric Ophthalmology for Pediatricians. Baltimore: Williams \& Wilkins; 1999. p. 39.

36. American Academy of Pediatrics Section on OphthaImology, American Association for Pediatric Ophthalmology and Strabismus, American Academy of Ophthalmology, American Association of Certified Orthoptists. Policy Statement. Red reflex examination in neonates, infants, and children. Pediatrics. 2008;122(6):1401-4. DOI 10.1542/peds.2008-2624.

37. Dobson V, Clifford-Donaldson CE, Miller JM, Garvey KA, Harvey EM. A comparison of Lea Symbol vs ETDRS letter distance visual acuity in a population of young children with a high prevalence of astigmatism. J AAPOS. 2009 Jun;13(3):253-7. DOI 10.1016/j.jaapos.2009.01.007.

38. O'Neill S, McAndrew DJ. The validity of visual acuity assessment using mobile technology devices in the primary care setting. Aust Fam Physician. 2016 Apr;45(4):212-5.

39. Zhang ZT, Zhang SC, Huang XG, Liang LY. A pilot trial of the iPad tablet computer as a portable device for visual acuity testing. J Telemed Telecare. 2013 Jan;19(1):55-9. DOI 10.1177/1357633X12474964.

40. Taub MB. Comprehensive examination procedures. In Taub MB, Bartuccio M, Maino DM. Visual diagnosis and care of the patient with special needs. Philadelphia: WoIters Kluwer/Lippincott Williams \& Wilkins; 2012. p. 157.

41. Morale SE, Hughbanks-Wheaton DK, Cheng C, Subramanian $\nabla$, O'Connor AR, Birch EE. Visual acuity assessment of children with special needs. Am Orthopt J. 2012;62:90-8. DOI 10.3368/aoj.62.1.90.

42. Nielsen LS, Skov L, Jensen H. Visual dysfunctions and ocular disorders in children with developmental delay. I. prevalence, diagnoses and aetiology of visual impairment. Acta Ophthalmol Scand. 2007 Mar;85(2):149-56. DOI 10.1111/j.1600-0420.2006.00867.x.

43. Kozeis N, Anogeianaki A, Mitova DT, Anogianakis G, Mitor T, Klisarova A. Visual function and visual perception in cerebral palsied children. Ophthalmic Physiol Opt. 2007 Jan;27(1):44-53. DOI 10.1111/j.14751313.2006.00413.x

44. Friedman NJ, Kaiser PK. The Massachusetts Eye and Ear Infirmary Illustrated Manual of Ophthalmology. $3^{\mathrm{a}}$ ed. Barcelona: EIsevier, 2010.

45. Wilson FM, Blomquist PH. Ocular motility examination. In: Practical Ophthalmology: A manual for beginning residents. 6th ed. San Francisco CA: American Academy of Ophthalmology; 2009. p. 93-4.

46. Guerrero JJ. Optometría clínica. $2^{a}$ ed. Bogotá: Fundación Universitaria del Área Andina; 2012.

47. Childrens Eye Foundation. Amblyopia Economic Costs: Diminished Utility [Internet]. [cited 2018]. Available from: https://www.childrenseyefoundation.org/S/ why-save-sight/economic-cost-of-failure-to-preventblindness-from-amblyopia/

48. Wittenborn J, Rein D. Cost of vision problems: The economic burden of vision loss and eye disorders in the United States. Chicago IL: NORC;2013 [cited 2013]. Available from: https://www researchgate.net/publication/249960785_Cost_of_Vision_Problems_The_Economic_Burden_of_Vision_Loss_and_Eye_Disorders in_the_United_States

49. Membreno JH, Brown MM, Brown GC, Sharma S, Beauchamp GR. A cost-utility analysis of therapy for ambIyopia. Ophthalmology. 2002 Dec;109(12):2265-71.

50. Wallace DK, Repka MX, Lee KA, Melia M, Christiansen SP, Morse CL. Amblyopia Preferred Practice Pattern. Ophthalmology. 2018 Jan;125(1):105-42. DOI 10.1016/j. ophtha.2017.10.008. 